\title{
A New Numerical Method for the Simulation of Three-Dimensional Flow in a Pipe
}

A. Leonard and A. Wray

July 1982

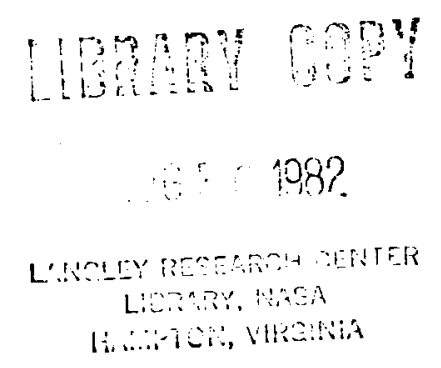


4 
ENTER:

3

1 FWMGS-Th-84267

DISPLY OSBA

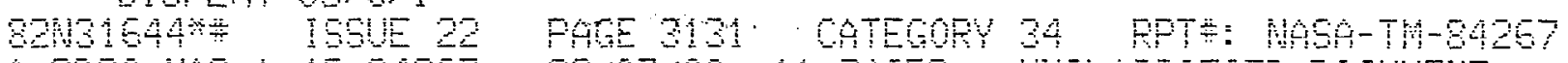

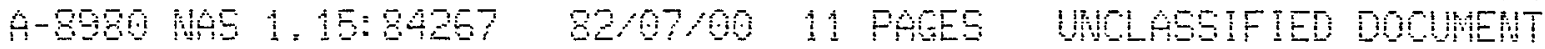

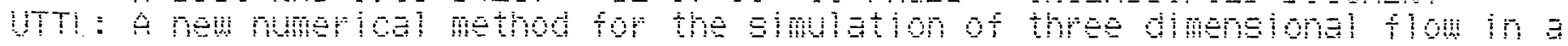
i ipe

AUTH: ALEOMAR, A. BARAY A.

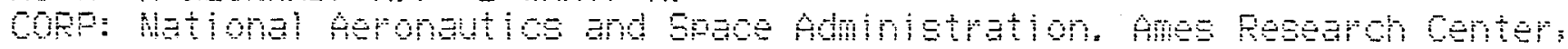

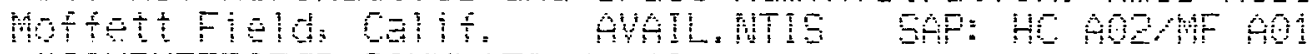

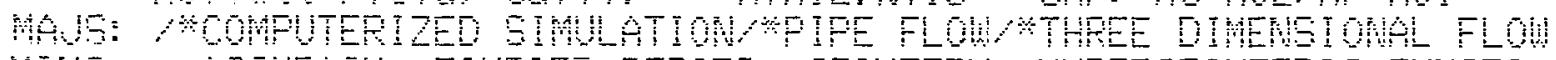

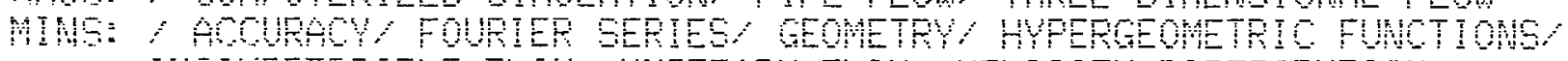

ABA: R.JF. 



\section{A New Numerical Method for the Simulation of Three-Dimensional Flow in a Pipe}

A. Leonard

A. Wray, Ames Research Center, Moffett Field, California

\section{NASก}

National Aeronautics and

Space Administration

Ames Research Center

Moffett Field. California 94035 


\title{
A NEW NUMERICAL METHOD FOR THE SIMULATION OF THREE-DIMENSIONAL
}

FLOW IN A PIPE

\author{
A. Leonard and A. Wray \\ NASA Ames Research Center \\ Moffett Field, CA 94035 U.S.A.
}

\section{INTRODUCTION}

In the last few years, major advances have been made in the numerical simulation of wall-bounded transitional and turbulent shear flows. So far, most of the emphasis has been on flows with planar boundaries. Transitional flows in a flatplate boundary layer (ref. 1) and in a channel (ref. 2), as well as turbulent channel flow (ref. 3), have been investigated without modeling the near-wall region; Moin (ref. 4) gives a critique of these investigations. Except for the study of Patera and Orszag (ref. 5) of axisymmetric pipe flow and the simulation of turbulent flow in annuli by Schumann (ref. 6), using modeled boundary conditions, very little work has been reported on nonplanar flows.

In this paper we present a new numerical technique for simulating threedimensional, unsteady, incompressible pipe flows and demonstrate its utility and accuracy. Each vector function in the expansion of the velocity field is divergencefree and satisfies the boundary conditions for viscous flow. Some of the benefits of the expansion technique are as follows: (1) pressure is eliminated from the dynamics, (2) only two unknowns per "mesh point" are required, (3) it provides implicit treatment of the viscous terms at no extra computational cost, and (4) no fractional time-steps are required.

In addition, the method uses spectral expansions: Fourier series in the azimuthal $(\theta)$ and streamwise $(x)$ directions and global polynomials in the radial $(r)$ direction. Thus, for smooth velocity fields, we expect rapid convergence of our expansions, independent of boundary constraints, as long as the radial polynomials are eigenfunctions of a singular Sturm-Liouville problem (ref. 7). In general, Chebychev or Legendre polynomials are good candidates, but we show that for cylindrical geometry a certain choice of the Jacobi polynomials is particularly advantageous in minimizing the coupling of the resulting equations for the expansion coefficients while satisfying the analytical behavior of the flow variables near $r=0$. As discussed below, the method has been tested on the linear stability problem for Poiseuille flow. 
MATHEMATICAL FOUNDATIONS

The governing equations are the incompressible Navier-Stokes equations for the velocity $\underline{u}$ and the disturbance pressure $\mathrm{p}$,

$$
\begin{gathered}
\frac{\partial \underline{u}}{\partial t}+\underline{\omega} x \underline{u}=-\nabla\left(p+\frac{1}{2} u^{2}\right)-\frac{d P}{d x} \hat{e}_{x}+\frac{1}{\operatorname{Re}} \nabla^{2} \underline{u} \\
\nabla \cdot \underline{u}=0 .
\end{gathered}
$$

Here $\underline{\omega}=\nabla x \underline{u}$ is the vorticity, Re is the Reynolds number, $\frac{\mathrm{dP}}{\mathrm{dx}}$ is the constant mean pressure gradient, and the density $\rho=1$ everywhere. The boundary condition at the pipe wall $(r=1)$ is $\left.\underline{u}\right|_{r=1}=0$. We assume periodic boundary conditions in the $\mathrm{x}$ direction with period $\mathrm{L}$.

We proceed formally by defining the projection operator $\mathscr{P}$ which projects an arbitrary vector field into the space of divergence-free fields satisfying tangency at the boundary; that is, if $\underline{\Omega}$ is an arbitrary vector field, then we have the unique decomposition,

$$
\underline{\Omega}=\underline{\psi}+\nabla \varphi
$$

where $\underline{\psi}$ satisfies

$$
\nabla \cdot \underline{\Psi}=0 \quad,\left.\quad \underline{\psi} \cdot \underline{\mathrm{n}}\right|_{\text {boundary }}=0
$$

and $\mathscr{P}$ is the operator that accomplishes this decomposition,

$$
\mathscr{P} \underline{\Omega}=\underline{\psi}
$$

By applying this operator to the momentum equation, we obtain the time-evolution of the divergence-free velocity field as (ref. 8)

$$
\frac{\partial \underline{u}}{\partial t}+\frac{1}{\operatorname{Re}} \mathscr{P}(\nabla \times \nabla \times \underline{u})=-\mathscr{P}(\underline{\omega} \times \underline{u})-\frac{\mathrm{d} P}{\mathrm{~d} x} \hat{\mathrm{e}}_{\mathrm{x}}
$$

Our overall strategy is to expand $\underline{\mathbf{u}}$ in terms of divergence-free vector functions satisfying the viscous boundary conditions and periodicity in $x$ and $\theta$. We then substitute this expansion into equation ( 1 ) and apply a weighted residual method which mimics the application of the projection operator to obtain evolution equations for the expansion coefficients. 
EXPANSION METHOD

We write the velocity field $\underline{u}$ as the expansion

$$
\underline{u}(r, \theta, x, t)=\sum_{n, k, l} a_{n, k ; l}(t) \underline{x}_{n}(r) \exp (i k x+i \ell \theta)
$$

where each expansion vector satisfies

$$
\nabla \cdot\left[x_{n}(r) \exp (i k x+i \ell \theta)\right]=0
$$

and

$$
\underline{x}_{n}(1)=0
$$

We derive a system of ordinary differential equations (ODE's) for the coefficients $a_{n, k, l}$ by substituting the above expansion into the momentum equation and taking the inner product of the result with a set of weight vectors which are divergencefree,

$$
\nabla \cdot\left[\underline{\xi}_{m}(r) \exp (-i k x-i \ell \theta)\right]=0
$$

and satisfy the inviscid boundary condition,

$$
\xi_{m}(1) \cdot \hat{e}_{r}=0
$$

[The $(k, l)$ dependence of $\underline{x}_{n}$ and $\underline{\xi}_{m}$ is suppressed.] This weighted residual method mimics the application of the projection operator. For example if $\underline{\zeta}=\underline{\xi}_{m}(r) \exp (-i k x-i \ell \theta)$ then

$$
\begin{aligned}
\int_{V} \underline{\zeta} \cdot \nabla \varphi \mathrm{dV} & =-\int_{V}(\nabla \cdot \underline{\zeta}) \varphi \mathrm{dV}+\int_{S} \varphi(\underline{\zeta} \cdot \underline{\mathfrak{n}}) \mathrm{d} S \\
& =0
\end{aligned}
$$

for an arbitrary scalar field $\varphi$ with periodic boundary conditions in $x$.

The result for each wave vector $(k, l)$ is a system of ODE's

$$
A \underline{\dot{a}}+\frac{1}{\operatorname{Re}} B \underline{a}=\underline{f}
$$

where

$$
A_{m n}=\int_{0}^{1} \xi_{m} \cdot \underline{x}_{n} r d r
$$




$$
\begin{aligned}
& B_{m n}=\int_{0}^{1} \xi_{m} \cdot \widetilde{\nabla \times \nabla} \times \underline{x}_{n} r d r \\
& f_{m}=-\int_{0}^{1} \underline{\xi}_{m} \cdot\left[\widetilde{\omega} \underline{\underline{u}}+2 \pi L \frac{d P}{d x} \hat{e}_{x}\right] r d r
\end{aligned}
$$

and where $\sim$ denotes double-Fourier transformation in $x$ and $\theta$. Thus, except for the nonlinear term, the coupling of the equations occurs only through the radial modes.

The choice of radial functions in $\underline{x}_{n}$ and $\xi_{m}$ must be made carefully to (1) minimize the coupling between radial modes (that is, obtain a banded structure for $A$ and $B$ if possible); (2) allow construction of $A$ and $B$ with relative ease; (3) obtain efficient computation of $\underline{\underline{f}}$; and (4) obtain rapid convergence while satisfying the constraints imposed on $\underline{x}_{n}$ and $\xi_{n}$, including the correct behavior as $r \rightarrow 0$.

We find that the sequence of expansion vectors $\left\{\underline{x}_{-1}, x_{0}^{+}, x_{0}^{-}, \ldots, x_{n}^{+}, x_{n}^{-}, \ldots\right\}$ defined in the following satisfies the above requirements. For $n \geqslant 0$ the $(r, \theta, x)$ components are

$$
\underline{x}_{n}^{ \pm}=\left(\begin{array}{c}
x_{n, r}^{ \pm} \\
x_{n, \theta}^{ \pm} \\
x_{n, x}^{ \pm}
\end{array}\right)=\left(\begin{array}{c} 
\pm i k q_{n}^{\ell \pm 1} \\
k q_{n}^{\ell \pm 1} \\
\mp \frac{1}{r} \frac{d}{d r}\left(r q_{n}^{\ell \pm 1}\right)-\frac{\ell}{r} q_{n}^{\ell \pm 1}
\end{array}\right)(k \neq 0)
$$

where

$$
q_{n}^{\ell}=r^{|\ell|}\left(1-r^{2}\right)^{2} g_{n}^{(|\ell|)}\left(r^{2}\right)
$$

and $g_{n}^{(l)}(y)$ is the shifted Jacobi polynomial (ref. 9),

$$
g_{n}^{(l)}(y)=P_{n}^{(o, l)}(2 y-1)
$$

satisfying the orthogonality condition

$$
\int_{0}^{l} y^{\ell} g_{m}^{(l)}(y) g_{n}^{(l)}(y) d y=c_{m}^{l} \delta, n
$$


For the case $k=0$, the above expansion vectors clearly are not complete and must be replaced by an alternative set. A convenient choice is given by $(n \geqslant 0)$

$$
\underline{x}_{n}^{-}=\left(\begin{array}{c}
-\frac{i \ell q_{n}^{\ell}}{r} \\
\frac{d q_{n}^{l}}{d r} \\
0
\end{array}\right) \underline{x}_{n}^{+}=\left(\begin{array}{c}
0 \\
0 \\
q_{n}^{\ell}
\end{array}\right)
$$

It is a simple matter to verify that the expansion vectors defined above yield the correct behavior of $\underline{\tilde{u}}(r, k, l)$ as $r \rightarrow 0$; for example, if $\ell>0$,

$$
\begin{aligned}
& \tilde{u}_{r} \rightarrow \gamma r^{\ell-1} \\
& \tilde{u}_{\theta} \rightarrow i \gamma r^{\ell-1} \\
& \tilde{u}_{x} \rightarrow \beta r^{\ell}
\end{aligned}
$$

where $\gamma$ and $\beta$ are complex constants. The additional vector $\underline{x}_{-1}$ is required because $\tilde{u}_{\theta}(k \neq 0)$ and $\tilde{u}_{x}(k=0)$ would otherwise have a double zero at the wall.

The corresponding weight vectors are essentially the curl of the $\underline{x}^{\prime} s$. More specifically, if $k \neq 0$ the weight vectors may be expressed as

$$
\overbrace{m}^{ \pm}=\nabla \times \nabla \times\left(\begin{array}{c}
\mp i q_{n}^{\ell \pm 1} \\
q_{n}^{\ell \pm 1} \\
0
\end{array}\right)
$$

while the $\underline{x}_{n}^{ \pm}$have the form

$$
\underline{x}_{n}^{ \pm}=\tilde{\nabla x}\left(\begin{array}{c}
-i q_{n}^{\ell \pm 1} \\
\mp q_{n}^{\ell \pm 1} \\
0
\end{array}\right)
$$

As a result, the $(+)$ vectors are uncoupled from the $(-)$ vectors. The resulting matrices A and B are nonadiagonal, except for an additional nonzero row and a column owing to the vector $\underline{x}_{-1}$. The limited bandwidth of the viscous matrix $B$ results from the particular choice of the polynomials $g_{n}^{(l)}$ given by 
equation (17). In particular, the Laplacian operator in the $(r, \theta)$ plane is equivalent to a tridiagonal matrix in the following sense:

$$
\begin{aligned}
\nabla^{2}\left[q_{n}^{\ell}(r) \exp (i \ell \theta]\right. & =\left(\frac{1}{r} \frac{d}{d r} r \frac{d}{d r}-\frac{\ell^{2}}{r^{2}}\right) q_{n}^{\ell}(r) \exp (i \ell \theta) \\
& =r^{\ell}\left[b_{n}^{l} g_{n-1}^{(l)}\left(r^{2}\right)+c_{n}^{\ell} g_{n}^{(l)}\left(r^{2}\right)+d_{n}^{l} g_{n}^{(l)}\left(r^{2}\right)\right] \exp (i \ell \theta)
\end{aligned}
$$

CONVERGENCE TESTS

As a test, the method described above was applied to the problem of determining the time eigenvalues for linearized flow in a pipe. The calculations were performed on a CDC 7600 computer. We assume $u \sim \exp (\lambda t)$ and order the eigenvalues such that $\operatorname{Rea} 1\left(\lambda_{1}\right) \geqslant \operatorname{Real}\left(\lambda_{2}\right) \geqslant \ldots$ The results for $\lambda_{1}$ with $\operatorname{Re}=9600, l=1, k=1$, are given in the table below, where $\mathrm{N}+2$ is the number of radial modes in the expansion.

\begin{tabular}{lll}
$\underline{N}$ & \multicolumn{2}{c}{$\lambda$} \\
\cline { 2 - 3 } 20 & -0.02312 & $-i 0.95050$. \\
25 & -0.02317074 & $-i 0.95048142$ \\
30 & $-0.023170795769-i 0.950481396659$ \\
35 & $-0.023170795764-i$ & 0.950481396668.
\end{tabular}

Note that the convergence is exponential in $N$ or some power of $N$, typical of spectral methods (ref. 7), and that there is no indication of significant round-off errors. The results agree with that of Salwen et al. (ref. 10), who obtained $\lambda_{1}=0.02317-i(0.95048)$, using an expansion in Stokes' eigenfunctions to solve the linear stability problem.

In figure 1 we show convergence of some of the higher eigenvalues for the case $k=1, \ell=1, R e=3000$, a Reynolds number in the range where a number of interesting transitional phenomena have been observed experimentally. Note that a large number of eigenvalues are predicted accurately for 30 to 35 radial expansion functions. In figure 2, the amplitudes of the coefficients of $\underline{x}_{n}^{-}$for the eigenvalue $\lambda_{1}$ are shown as a function of $n$ for four Reynolds numbers with $k=1$ and $\ell=1$. Each of the ${\underline{\chi_{n}}}_{n}^{-}$are normalized so that

$$
\int_{0}^{1}\left|\underline{x}_{n}^{-}\right|^{2} r d r=1
$$


all Ih, radial expansion $\mathrm{N}=35$ (37 expansion functions) was used for all cases. ly:till, the coefficients approach zero exponentially in some power of $n$.

SIIMMMRY

A new numerical method has been developed to investigate three-dimensional, unsteady fipe flows using a new velocity-vector expansion method. Each vector function in the expansion set is divergence-free and satisfies the boundary conditions for viscous flow. Other features of the general technique are as follows: (1) pressure is eliminated from the dynamics; (2) only two unknowns per "mesh point" are required; (3) there is rapid convergence of spectral methods; (4) there is implicit treatment of the viscous term at no extra computational cost; and (5) no fractional time-steps are required. In the present application of the method to flow in a pipe, the behavior of each flow variable near the computational singular point is treated rigorously and expansions in Jacobi polynomials have been shown to be particularly advantageous. The method has been tested on the linear stability problem for Poiseuille flow and has demonstrated rapid convergence of the eigenvalues and eigenfunctions as the number of radial modes is increased.

ACKNOWLDEGMENT

The authors wish to thank Dr. Parviz Moin and Mr. Robert Moser for many helpful discussions.

\section{REFERENCES}

1. Wray, A., and Hussaini, Y.: Numerical Experiments in Boundary Layer Stability, AIAA Paper 80-0275, Pasadena, Calif., 1980.

2. Orszag, S. A., and Kells, L. C.: Transition to Turbulence in Plane Poiseuille and Plane Couette Flow, J. Fluid Mech., Vol. 96, 1980, p. 159.

3. Moin, P., and Kim, J.: Numerical Investigation of Turbulent Channel Flow, J. Fluid Mech., Vol. 118, 1982, p. 341.

4. Moin, P.: Numerical Simulation of Wa11-Bounded Turbulent Shear Flows, Proceedings 8th International Conference on Numerical Methods in Fluid Dynamics (this issue).

5. Patera, A. T., and Orszag, S. A.: Finite-Amplitude Stability of Axisymmetric Pipe Flow, J. Fluid Mech., Vol. 112, 1981, p. 467.

6. Schumann, U.: Subgrid Scale Model for Finite Difference Simulations of Turbulent Flows in Plane Channels and Annuli, J. Comp. Phys., Vol. 18, 1975, p. 376. 
7. Orszag, S. A.: Spectral Methods for Problems in Complex Geometries, J. Comp. Phys., Vo1. 37, 1980, p. 70.

8. Chorin, A. J., and Marsden, J. E.: A Mathematical Introduction to Fluid Mechanics. Springer-Verlag, New York $\frac{1}{1979 .}$

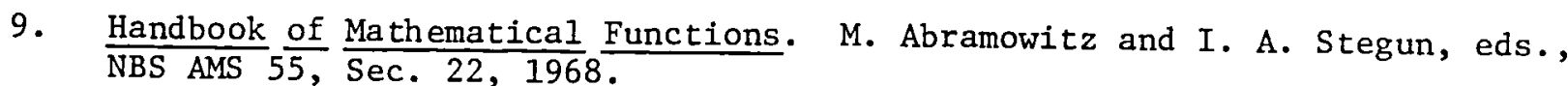

10. Salwen, H., Cotton, F. W., and Grosch, C. E.: Linear Stability of Poiseuille Flow in a Circular Pipe, J. Fluid Mech., Vol. 98, 1980, p. 273.

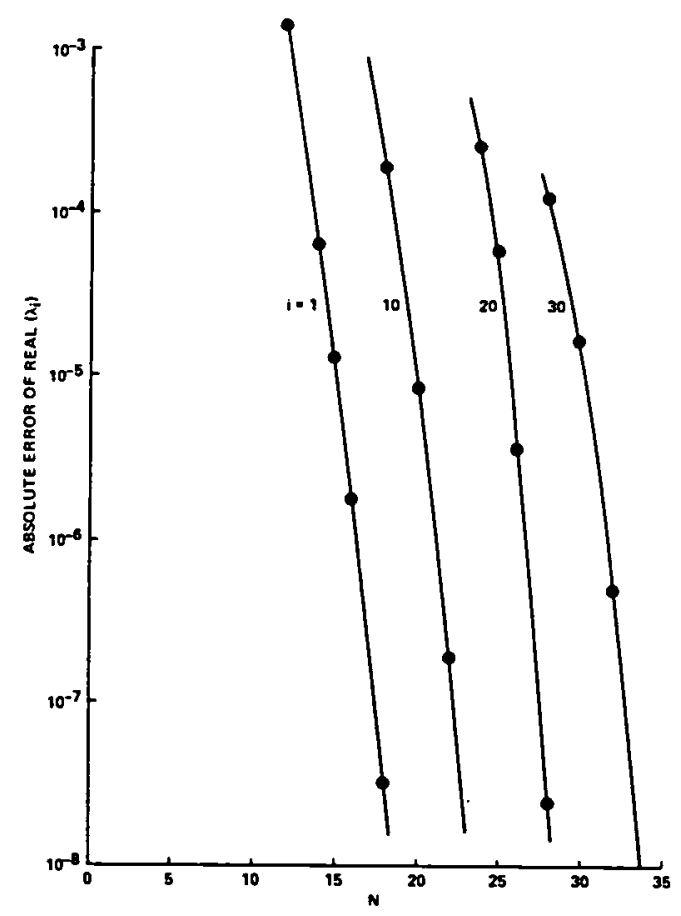

Fig. 1. Convergence of $\operatorname{Real}\left(\lambda_{i}\right) ; \mathrm{k}=\ell=1, \operatorname{Re}=3000$.

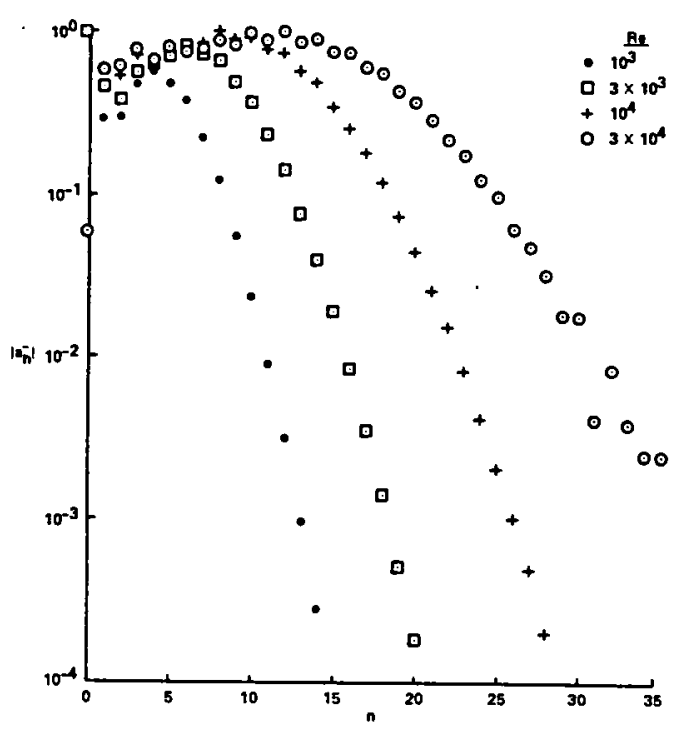

Fig. 2. Amplitudes of the expansion coefficients for the eigenvector corresponding to $\lambda_{l} ; k=\ell=1$. 


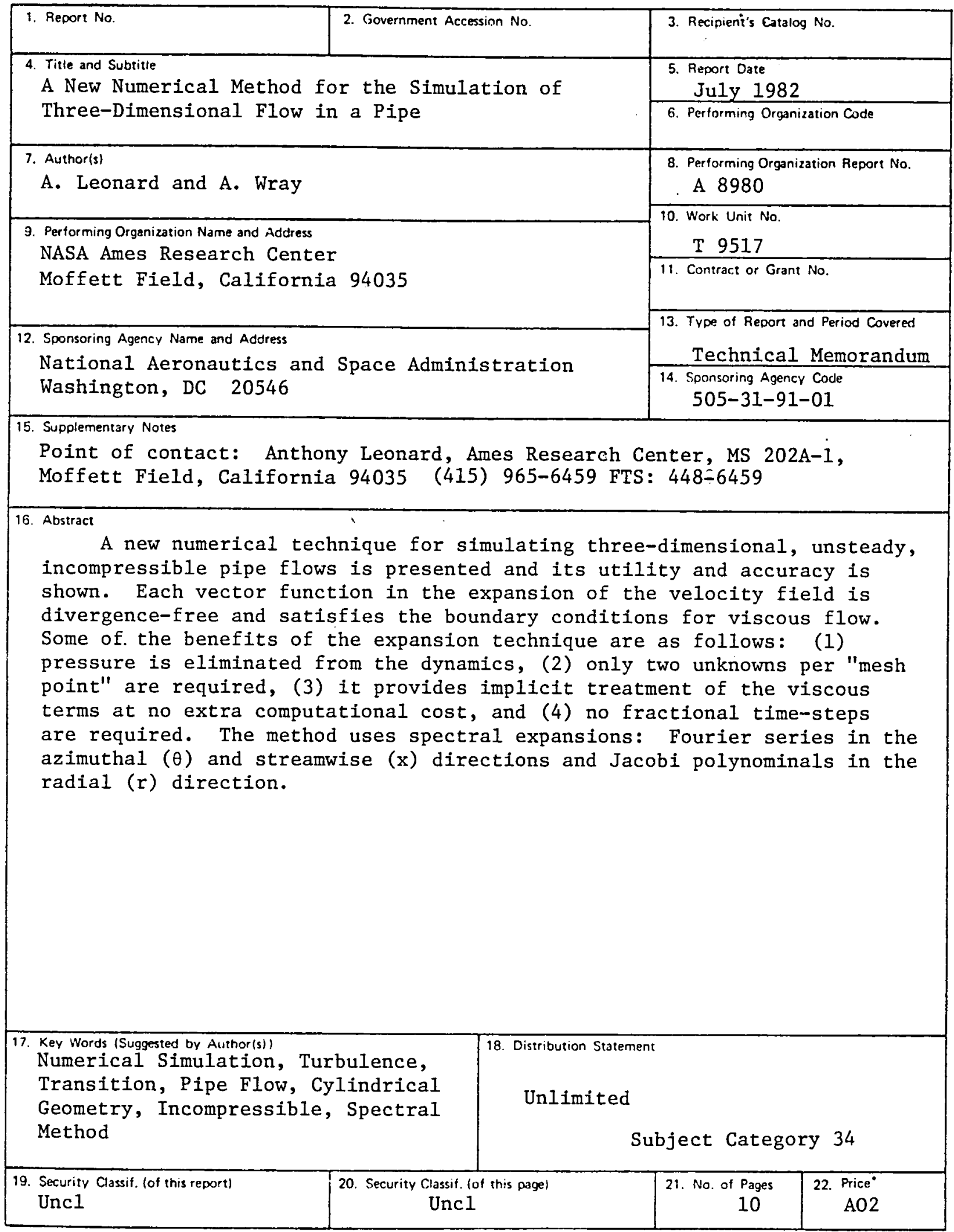

*For sale by the National Trennical Information Service, Springfield, Virgma 22161 

